\title{
Current State of Target Treatment in BRAF Mutated Melanoma
}

\author{
Enrica Teresa Tanda ${ }^{1 \dagger}$, Irene Vanni ${ }^{2,3 \dagger}$, Andrea Boutros ${ }^{1}$, Virginia Andreotti ${ }^{2,3}$, \\ William Bruno ${ }^{2,3}$, Paola Ghiorzo ${ }^{2,3 *}$ and Francesco Spagnolo ${ }^{1}$ \\ ${ }^{1}$ Medical Oncology, IRCCS Ospedale Policlinico San Martino, Genoa, Italy, ${ }^{2}$ Genetics of Rare Cancers, IRCCS Ospedale \\ Policlinico San Martino, Genoa, Italy, ${ }^{3}$ Genetics of Rare Cancers, Department of Internal Medicine and Medical Specialties, \\ University of Genoa, Genoa, Italy
}

\section{OPEN ACCESS}

Edited by:

Cristina Pellegrini,

University of L'Aquila, Italy

Reviewed by:

José Manuel Lopes, University of Porto, Portugal Jorge Lima,

University of Porto, Portugal

${ }^{*}$ Correspondence:

Paola Ghiorzo

paola.ghiorzo@unige.it

${ }^{t}$ These authors have contributed equally to this work

Specialty section:

This article was submitted to

Molecular Diagnostics

and Therapeutics,

a section of the journal

Frontiers in Molecular Biosciences

Received: 23 April 2020

Accepted: 19 June 2020

Published: 14 July 2020

Citation:

Tanda ET, Vanni I, Boutros A, Andreotti V, Bruno W, Ghiorzo P and Spagnolo F (2020) Current State of Target Treatment in BRAF Mutated Melanoma. Front. Mol. Biosci. 7:154. doi: 10.3389/fmolb.2020.00154
Incidence of melanoma has been constantly growing during the last decades. Although most of the new diagnoses are represented by thin melanomas, the number of melanoma-related deaths in 2018 was 60,712 worldwide (Global Cancer Observatory, 2019). Until 2011, no systemic therapy showed to improve survival in patients with advanced or metastatic melanoma. At that time, standard of care was chemotherapy, with very limited results. The identification of BRAF V600 mutation, and the subsequent introduction of BRAF targeting drugs, radically changed the clinical practice and dramatically improved outcomes. In this review, we will retrace the development of molecular-target drugs and the current therapeutic scenario for patients with BRAF mutated melanoma, from the introduction of $B R A F$ inhibitors as single agents to modern clinical practice. We will also discuss the resistance mechanisms identified so far, and the future therapeutic perspectives in BRAF mutated melanoma.

Keywords: melanoma, BRAF mutation, targeted therapy, MAPK pathway, metastatic disease

\section{INTRODUCTION}

Cutaneous melanoma has one of the highest mutational rate among all solid tumors (The Cancer Genome Atlas Network, 2015). Some of these mutations involve specific oncogenes, causing alterations in cell cycle regulation, proliferation and apoptosis. Multiple molecular pathways are implicated: among these, one of the most characterized is the Mitogen-Activated Protein Kinase (MAPK). This molecular pathway is composed by a Tyrosine Kinases Receptor (TKR), RAS, RAF, MEK and ERK proteins (Figure 1). Simplifying, the binding between a growth factor and the TKR leads to a phosphorylation cascade resulting in the activation of ERK. ERK, in turn, regulates the expression of many genes involved in cell proliferation and survival (Gaestel, 2006). The mutation of a gene coding for one of these proteins can constitutively activate the whole pathway.

Activating $B R A F$ mutation occurs in approximately $50 \%$ of cutaneous melanoma (The Cancer Genome Atlas Network, 2015; Sanchez-Vega et al., 2018). To date, about 300 BRAF mutations have been characterized, the most common being the V600E (valine to glutamic acid; 7088\%) (Rubinstein et al., 2010; Lovly et al., 2012; Menzies et al., 2012). The identification and characterization of $B R A F$ mutations led to the development of highly specific drugs which radically changed the therapeutic landscape of melanoma. Indeed, targeted therapies substantially improved survival in patients with advanced or metastatic melanoma from a median of 6 months obtained with chemotherapy (Korn et al., 2008), the standard of care before the approval of the first BRAF inhibitor, to a median of 25.9-33.6 months (Robert et al., 2019; Ascierto et al., 2020). Moreover, 


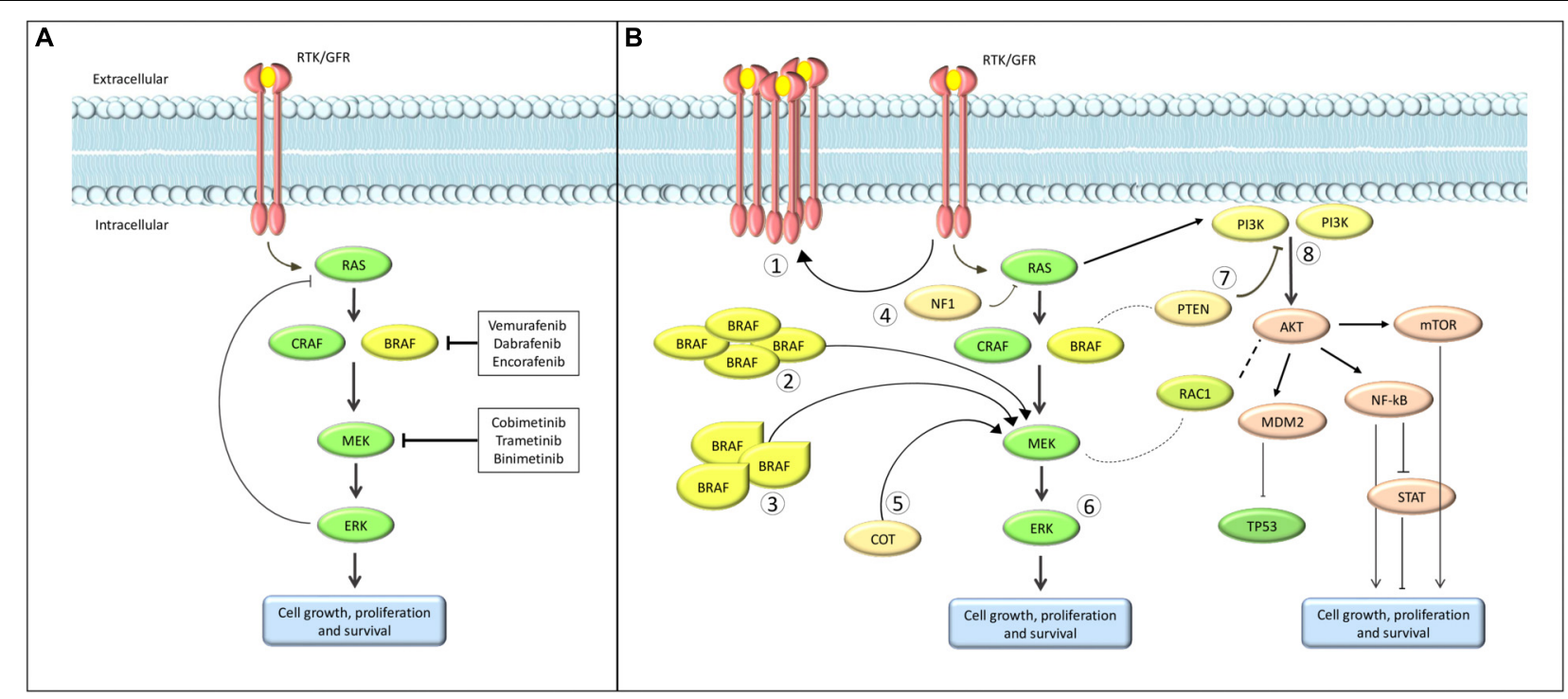

FIGURE 1 | Schematic overview of the MAPK pathway. (A) normal pathway; (B) the most common resistance mechanisms. (1) Upregulation of RTK. (2) BRAF amplification. (3) BRAF alterantive splicing. (4) Loss of NF1. (5) COT overexpression. (6) ERK activation. (7) Loss of PTEN. (8) Alternative pathways activation.

targeted therapies showed a significant benefit in the adjuvant setting with a $53 \%$ decrease in the risk of relapse compared with placebo (Long et al., 2017b). These results recently lead to the approval of BRAF plus MEK inhibitors for high risk resected (stage III) melanoma patients (Long et al., 2017b; Spagnolo et al., 2019). These revolutionary changes underline the importance of the early molecular characterization of highrisk stage II, stage III and IV melanoma patients, which has become mandatory according to the ESMO Clinical Practice Guidelines (Michielin et al., 2019) and represents a fundamental step for personalized therapy. For this reason, the assessment of $B R A F$ mutations nowadays constitutes a fundamental diagnostic procedure and essential in the current clinical practice of oncology. The molecular biology-based strategies used for BRAF mutation detection have been extensively described in a related review (Vanni et al., 2020).

In this review we will retrace the development of moleculartarget drugs and the current therapeutic scenario for patients with $B R A F$ mutated melanoma, from the introduction of BRAF inhibitors as single agents in 2011 to modern clinical practice. We will also discuss the resistance mechanisms identified so far, and the future therapeutic perspectives in BRAF mutated melanoma.

\section{BRAF Inhibitors}

The first drug used as BRAF inhibitor in patients with $B R A F$ V600E advanced or metastatic melanoma was sorafenib (BAY 43-9006), which showed promising results in murine models but failed the human experimentation (Eisen et al., 2006; Hauschild et al., 2009).

In 2005 and later in 2009, BRAF inhibitors PLX4032 (vemurafenib) and GSK2118436 (dabrafenib) were synthesized. Finally, in 2013 LGX818, or encorafenib, began clinical investigation.
In the randomized phase 3 studies BRIM-3 (Chapman et al., 2011, 2017; McArthur et al., 2014) and BREAK-3 (Hauschild et al., 2012; Latimer et al., 2015), BRAF inhibitors vemurafenib and dabrafenib, respectively, obtained a statistically significant benefit in terms of overall survival (OS), progression-free survival (PFS) and overall response rate (ORR) compared to chemotherapy (Table 1).

These results fueled molecular targeted drug research and raised several new issues.

First, the problem of resistance. Indeed, about $15 \%$ of patients showed no response to BRAF inhibition (Spagnolo et al., 2015) and, among responders, about $50 \%$ developed acquired resistance after a median of 6-8 months.

Another challenge was the paradoxical effect of BRAF inhibitors on BRAF-wild type cells.

Safety analysis of BRIM-3 study showed that about $20 \%$ of patients developed cutaneous squamous carcinoma. This phenomenon is due to a collateral activation of the MAPK pathway in BRAF-wild type keratinocytes (Heidorn et al., 2010; Poulikakos et al., 2010; Su et al., 2012). The mechanism behind this paradoxical effect is not entirely clear but it seems related to conformational change in wild-type BRAF protein, CRAF dimerization and ERK activation. This process might also induce new malignant melanoma (Zimmer et al., 2012; Dalle et al., 2013), RAS-mutant leukemia (Callahan et al., 2012) and other second neoplasms (Gibney et al., 2013).

\section{BRAF Inhibitors Plus MEK Inhibitors}

Preclinical studies suggested that the addition of a MEK inhibitor to a BRAF inhibitor could reduce the side effects of BRAF inhibitor as single agent (e.g., paradoxical effect), delay the development of resistance and generate a synergistic improvement in efficacy outcomes (King et al., 2013). On this 
TABLE 1 | Summary of selected targeted therapy trials in BRAF-mutant advanced melanoma.

\begin{tabular}{|c|c|c|c|c|c|c|}
\hline Trial & Drugs & & Median OS (mo) & Median PFS (mo) & ORR & References \\
\hline \multirow[t]{2}{*}{ BRIM-3 } & Vemurafenib & & 13.6 & 6.9 & $57 \%$ & McArthur et al., 2014; Chapman et al., 2017 \\
\hline & Dacarbazine & & 9.7 & 1.6 & $9 \%$ & \\
\hline \multirow[t]{2}{*}{ BREAK-3 } & Dabrafenib & & 18.2 & 6.7 & $53 \%$ & Hauschild et al., 2012; Latimer et al., 2015 \\
\hline & Dacarbazine & & 15.6 & 2.9 & $6 \%$ & \\
\hline \multirow[t]{2}{*}{ Combi-D } & Dabrafenib + trametinib & & 25.1 & 11 & $69 \%$ & Long et al., 2015 \\
\hline & Dabrafenib & & 18.7 & 8.8 & $53 \%$ & \\
\hline \multirow[t]{2}{*}{ Combi-V } & Dabrafenib + trametinib & & 26.1 & 12.1 & $67 \%$ & Robert et al., 2016 \\
\hline & Vemurafenib & & 17.8 & 7.3 & $53 \%$ & \\
\hline \multirow[t]{2}{*}{ CoBRIM } & Vemurafenib + Cobimetinib & & 22.3 & 12.3 & $68 \%$ & Society for Melanoma Research Congress, 2019 \\
\hline & Vemurafenib & & 17.4 & 7.2 & $50 \%$ & \\
\hline \multirow[t]{3}{*}{ COLUMBUS } & Encorafenib + Binimetinib & & 33.6 & 14.9 & $64 \%$ & Ascierto et al., 2020 \\
\hline & Vemurafenib & & 16.9 & 7.3 & $41 \%$ & \\
\hline & Encorafenib & & 23.5 & 9.6 & $52 \%$ & \\
\hline Trial & Drugs & Cohorts & Median OS & Median PFS & $\begin{array}{l}\text { Intracranial response }(\%) \text { by } \\
\text { investigators - IRC* }\end{array}$ & References \\
\hline \multirow[t]{2}{*}{ BREAK - MB } & Dabrafenib & A (previously untreated BM) & 33.1 weeks & 16.1 weeks & $39.2 \%^{\star \star}-20 \%$ ** & Long et al., 2012 \\
\hline & & B (previously treated BM) & 31.4 weeks & 16.6 weeks & $30.8 \%^{* *}-19 \%^{* *}$ & \\
\hline \multirow[t]{2}{*}{ Vemurafenib } & Vemurafenib & 1 (previously untreated BM) & 8.9 month & 3.7 months & $29 \%-18 \%$ & Mcarthur et al., 2016 \\
\hline & & 2 (previously treated BM) & 9.6 month & 4 months & $23 \%-18 \%$ & \\
\hline \multirow[t]{4}{*}{$\mathrm{COMBI}-\mathrm{MB}$} & Dabrafenib + trametinib & $\begin{array}{l}\text { A (BRAF V600E asymptomatic, } \\
\text { previously untreated BM) }\end{array}$ & 10.8 & 5.6 & $58 \%$ & Davies et al., 2017 \\
\hline & & $\begin{array}{l}\text { B (BRAF V600E asymptomatic, } \\
\text { previously treated BM) }\end{array}$ & 24.3 & 7.2 & $56 \%$ & \\
\hline & & $\begin{array}{l}\mathrm{C}(\mathrm{BRAF} \text { V600D/K/R, } \\
\text { asymptomatic, previously } \\
\text { treated or untreated BM) }\end{array}$ & 10.1 & 4.2 & $44 \%$ & \\
\hline & & $\begin{array}{l}\mathrm{D}(\mathrm{BRAF} \text { V600D/ E/K/R, } \\
\text { symptomatic, previously treated } \\
\text { or untreated BM) }\end{array}$ & 11.5 & 5.5 & $59 \%$ & \\
\hline
\end{tabular}

*Independent review committee (IRC). ${ }^{*}$ Considering BRAF V6O0E-mutated patients. Abbreviations: BM, brain metastases; OS, overall survival, PFS, progression-free survival; ORR, overall response rate; IRC, independent review committee; MO, months. 
wave, several studies have been performed. Taken together (Pasquali et al., 2018) data from these clinical trials demonstrated a statistically significant superiority of the combination compared to monotherapy in terms of OS, PFS and ORR (Queirolo and Spagnolo, 2017; Table 1).

Dabrafenib plus Trametinib. The phase I/II trial (Flaherty et al., 2012) demonstrated the safety of dabrafenib and trametinib combination and its significant superiority in terms of ORR and PFS over dabrafenib monotherapy, among patients with BRAF V600E/K-mutated, unresectable or metastatic melanoma. Moreover, a subsequent survival analysis showed its advantage in terms of survival (Long et al., 2016). Based on these results, the combination of dabrafenib and trametinib received FDA approval in January 2014, with an accelerated procedure.

The phase III trial, COMBI-d (Long et al., 2014) compared dabrafenib plus trametinib with dabrafenib monotherapy. In the primary analysis, median PFS and ORR were significantly increased in the combination arm. A subsequent update (Long et al., 2015) demonstrated that the combination reduced the risk of death by $29 \%$ compared with monotherapy with a 3 years OS of 44 vs 32\% (Long et al., 2017a).

Combination of dabrafenib and trametinib was also tested in another phase III trial, the COMBI-v study (Robert et al., 2015), in comparison with vemurafenib. Data showed a significant benefit for the combination in terms of ORR, median PFS and median OS. Even better results were obtained in the population with normal lactate dehydrogenase $(\mathrm{LDH})$, in terms of median PFS [17.5 vs 9.2 months - Hazard Ratio (HR) 0.55] and survival (median OS not reached vs 21.5 months - HR 0.56) (Robert et al., 2016). Similar results, in terms of clinical activity, were observed in real world population, with an ORR of $67 \%$ (Atkinson et al., 2020).

The most recent update of these studies is a pooled analysis published in 2019 (Robert et al., 2019). Efficacy outcomes were confirmed to be completely superimposable between the two trials, underling the strength of the evidence, and showed a 5-year PFS of $19 \%$ and a 5 -year OS of $34 \%$.

Vemurafenib plus Cobimetinib. In the Phase Ib study BRIM-7 (Ribas et al., 2014), vemurafenib plus cobimetinib showed a significant benefit in terms of ORR, PFS and OS, and the results of the subsequent phase III trial, coBRIM (Larkin et al., 2014), led to the FDA registration of this combination. Indeed, combination therapy showed its superiority on the comparison arm (vemurafenib) in terms of both PFS (HR 0.51) and ORR. A subsequent analysis (Society for Melanoma Research 2016 Congress, 2017) showed a higher number of complete response (16 vs $11 \%$ ), which suggested that some patients may get a better response if treatment is continued. Final analysis has been presented at Society for Melanoma Research (SMR) congress 2019 (Society for Melanoma Research Congress, 2019), and confirmed the substantial superiority of the combination over monotherapy.

Encorafenib plus Binimetinib. In the phase III study COLUMBUS (Dummer et al., 2018), 577 patients received encorafenib (450 $\mathrm{mg}$ daily) plus binimetinib (45 mg twice daily) or encorafenib (300 mg) or vemurafenib (960 mg twice daily). The study was divided into two parts: the first one aimed to compare efficacy of the combination vs vemurafenib (primary endopoint) and encorafenib, while the second one aimed to characterize the contribute of binimetinib in the obtained outcome.

Results of part one showed the superiority of encorafenib plus binimetinib combination over vemurafenib in terms of ORR, PFS and OS. Analysis at 3 years showed an OS of $47 \%$ for the combination, $41 \%$ for encorafenib and $31 \%$ for vemurafenib, with a death risk reduction of 39\% (Ascierto et al., 2020). Also in this case, a recently published landmark analysis showed that the subgroups of patients with normal $\mathrm{LDH}$ and less than three metastatic sites were the ones with the best outcomes (Ascierto et al., 2020).

Part 2 of the study randomized 344 patients in a 3:1 ratio to receive either encorafenib $300 \mathrm{mg}$ plus binimetinib $45 \mathrm{mg}$ twice daily or encorafenib $300 \mathrm{mg}$. Results, presented at ESMO 2017 (Dummer et al., 2017), confirmed the superiority of combination treatment over monotherapy in terms of ORR, PFS and OS.

Safety. Globally, combination therapies are well tolerated and present an acceptable toxicity profile. Some adverse events are quite common in all combination schemes, such nausea, diarrhea, vomiting, fatigue, headache, arthralgia and a lower rate of cutaneous squamous cell carcinomas. Other adverse events are more frequent with specific combination, like pyrexia and chills for dabrafenib and trametinib, photosensitivity and diarrhea for vemurafenib and cobimetinib and laboratory alterations with encorafenib and binimetinib. Finally, dabrafenib and trametinib showed to significantly improve patients' quality of life compared to both dabrafenib and vemurafenib monotherapy (Grob et al., 2015; Schadendorf et al., 2015), while vemurafenib and cobimetinib showed to maintain the patient's quality of life compared with vemurafenib monotherapy, increasing efficacy (Dréno et al., 2018).

\section{Brain Metastasis}

Brain involvement is frequent in melanoma patients and is associated with poor prognosis (Davies et al., 2011; Spagnolo et al., 2016). About $20 \%$ of patients have brain metastases (BM) at the initial diagnosis of advanced disease, and more than $40 \%$ develop BM at some point of their disease course; up to $75 \%$ patients have BM in autopsy studies (Long and Margolin, 2020). Before the introduction of immunotherapy and target therapy, median OS of this group of patients was 3.8-5.0 months (Davies et al., 2011).

$B R A F$ inhibitors. Dabrafenib and vemurafenib were tested through two phase II trial. In both studies patients were divided into two cohorts: one cohort included patients naive for previous BM local treatment, while the second cohort included patients who progressed after BM local treatment (surgery, WBRT, or SRS). Both dabrafenib (BREAK-MB) (Long et al., 2012) and vemurafenib (Mcarthur et al., 2016) showed clinical activity in both cohorts in terms of intracranial ORR, PFS and OS (Table 1). Of note, dabrafenib obtained greater 
results among BRAF V600E-mutated than BRAF V600Kmutated patients. In both trials, the extracranial $O R$, the median DOR, the median PFS and OS were lower than that observed in previous studies with dabrafenib and vemurafenib, and these data support the hypothesis that BM in BRAFmutant melanoma are less responsive to BRAF inhibition, probably due to some different characteristics of $\mathrm{BM}$, or differences in drug concentrations between intracranial and extracranial compartments.

Combination of BRAF plus MEK inhibitors. In COMBI$\mathrm{MB}$, a phase II study, 125 BRAF V600-mutated melanoma patients with $B M$ were divided into four cohorts and treated with dabrafenib plus trametinib (Davies et al., 2017). Cohort A included 76 BRAF V600E-mutated, asymptomatic patients, naive for local brain therapy; cohort $\mathrm{B}$ included $16 B R A F$ V600E-mutated, asymptomatic patients, previously treated with local brain therapy; Cohort C included 16 BRAF V600D/K/Rmutated, asymptomatic patients, naive for local brain therapy or previously treated; Cohort D included 17 BRAF V600D/E/K/Rmutated, symptomatic patients, naive for local brain therapy or previously treated. The primary endpoint of the trial, the intracranial response in cohort $\mathrm{A}$, was met with a rate of $58 \%$ including a $4 \%$ of intracranial complete response, and a median duration of response of 6.5 months. Intracranial response was also seen in cohorts $\mathrm{B}, \mathrm{C}$ and $\mathrm{D}$ but these data should be considered exploratory, due to the sample size of the two cohorts. Response rate was lower than observed in patients with just extracranial disease (58 vs 67\%) and the median PFS was significantly shorter (5.6 vs 10.1 months), which suggests an earlier treatment failure in BM. Finally, another clinical trial with vemurafenib, cobimetinib and atezolizumab is currently ongoing and results are awaited (NCT03625141) (ClinicalTrials.gov, 2020).

\section{Adjuvant Setting}

Considering the extraordinary results obtained in the metastatic setting, studies on the efficacy of BRAF and MEK inhibitors in the adjuvant setting were initiated (Long et al., 2017b; Spagnolo et al., 2019).

In the BRIM-8 trial (Maio et al., 2018), patients diagnosed with stage IIC, IIIA, IIIB and IIIC BRAF-mutated melanoma were randomized to receive either vemurafenib or placebo. Patients were divided into two cohorts: cohort one included stage IIC, IIIA, IIIB, and cohort two included patients with IIIC disease. Results of the study showed that 1 year of adjuvant vemurafenib provided a substantial disease-free survival (DFS) benefit (46\% risk reduction vs placebo) in cohort one, while in cohort two increased median DFS, demonstrating a biologic effect, but did not statistically significantly reduce DFS risk.

In the phase III COMBI-AD trial (Long et al., 2017b), 870 patients diagnosed with IIIA (with lymph node metastasis $>1 \mathrm{~mm}$ ), IIIB and IIIC BRAF-mutated melanoma were treated with dabrafenib plus trametinib or placebo for 1 year. Primary endpoint was relapse-free survival (RFS), while OS and distant metastasis-free survival (DMFS) were exploratory endpoints. In October 2018, results with about 4 years of follow-up were published. Data showed a 4 -year RFS of $54 \%$ in the experimental arm vs $38 \%$ in the placebo arm (HR 0.49) with an estimated cure rate of 54 vs 37\%, respectively (Hauschild et al., 2018). Threeyear OS was 86 vs $77 \%$. Notably, the delta between the curves increased over time, suggesting a potential long-term impact on survival.

\section{Resistance and Beyond}

During treatment with BRAF plus MEK inhibitors, primary and acquired resistance remain a significant challenge (Figure 1). Several preclinical studies tried to understand mechanisms of resistance, with the aim of preventing or blocking them. Simplifying, it is possible to divide the most understood mechanisms of resistance between intracellular and extracellular mechanisms.

Among intracellular mechanisms, the most characterized include the reactivation of the MAPK signaling pathway with ERK activation (Nissan et al., 2013; González-Cao et al., 2015) or overexpression of TKR, and the activation of alternative intracellular molecular pathways (Figure 1).

Several ERK-inhibitors are currently in development (Carlino et al., 2014). GDC-0994, a highly selective ERK1/2 inhibitor, has demonstrated activity in combination with cobimetinib in preclinical models (Robarge et al., 2014). SCH772984 has shown promising results in a panel of melanoma cell lines, including cells with innate or acquired resistance to vemurafenib, cells with $B R A F / N R A S$ double mutations or NRAS mutations (Morris et al., 2013; Wong et al., 2014) (NCT02457793).

Blocking the MAPK pathway at the RTK level has a strong rationale, if we consider that RTK could activate simultaneously MAPK and alternative pathways like PI3K-AKT-mTOR (Nazarian et al., 2010; Villanueva et al., 2010; Straussman et al., 2012; Wilson et al., 2012): multi-RTK-inhibitors (e.g., lenvatinib), selective small-molecule RTK-inhibitors (capmatinib, BGJ398, and MEHD7945A), and monoclonal antibodies that bind the extracellular domain of the RTK (onartuzumab, ganitumab) are in course of study in combination with BRAF- or MEK-inhibitors (ClinicalTrials.gov, 2020). Moreover, numerous inhibitors that target various levels of the PI3K pathway are in development, including PI3K, AKT, and mTOR inhibitors (Shi et al., 2014; Manzano et al., 2016).

Regarding extracellular mechanisms, increasingly strong arguments support the importance of the tumor microenvironment and the modulation of the immune system.

These observations led to the develop of immune-checkpoints inhibitors, such ipilimumab, nivolumab, and pembrolizumab, monoclonal antibodies aimed to interact with CTLA-4 and PD1 to re-establish immune response against the tumor. All these molecules allowed to obtain durable responses and long-term survival in patients with advanced or metastatic melanoma. At the same time, several studies demonstrated that BRAF inhibitors can impact on immune responses in a direct (e.g., increase of intratumour infiltrating lymphocytes - TILs) and an indirect (increase of melanoma antigens expression) way suggesting a strong rationale of combining these two therapeutic strategies (Boni et al., 2010; Khalili et al., 2012; 
Koya et al., 2012; Frederick et al., 2013; Sapkota et al., 2013; Kakavand et al., 2015).

The first combination trials with ipilimumab and vemurafenib unfortunately demonstrated significant toxicities that limited further development (Ribas et al., 2013; Minor et al., 2015).

Combination strategies with BRAF inhibitors and anti-PD1/PD-L1 agents have also been tested.

First, the combination of vemurafenib plus atezolizumab (anti-PD-L1). Early data from this experimentation were presented at the SMR 2015 Congress and showed an ORR rate of $76 \%$, without unexpected adverse events being reported. Encouraged by these exciting results, this trial was expanded adding cobimetinib to vemurafenib and atezolizumab, reaching an ORR of 83\% (Society for Melanoma Research 2016 Congress, 2017). Based on these findings, a randomized phase III study with vemurafenib, cobimetinib and atezolizumab/placebo was initiated, but results are not available yet (NCT02908672) (ClinicalTrials.gov, 2020).

A similar approach with dabrafenib, trametinib and pembrolizumab was tested in the phase I (Ribas et al., 2016) and II trial KEYNOTE-022 (NCT02130466) (ClinicalTrials.gov, 2020), that compared the triple combination vs dabrafenib, trametinib and placebo. Data from the phase II part, presented during SMR Congress 2019 (Society for Melanoma Research Congress, 2019), with a median follow-up of 28 months, showed a median PFS (primary endpoint) of 16.9 months for the pembrolizumab plus dabrafenib and trametinib arm vs 10.7 months for placebo plus dabrafenib and trametinib arm (HR 0.53) with a 24-months PFS rates of 41 and $16.3 \%$. Unfortunately, these results were not statistically significant and the primary endpoint of the study was not met. Median OS in the triple combination arm was not reached vs 26.3 months in dabrafenib plus trametinib arm (HR 0.64), and OS rates at 24-months were 63 and 51.7\% respectively. Moreover, the duration of response was longer in the experimental arm.

Finally, acquired resistance could be also caused by epigenetic changes that may be reversible (Van Allen et al., 2014). For this reason, another interesting approach in melanoma patients is the rechallenge with targeted therapies, despite data from randomized trials are lacking. In a recently published minireview (Reschke et al., 2019), 238 patients from several retrospective and prospective trials were analyzed showing a disease control rate of $67 \%$.

\section{CONCLUSION}

In summary, targeted therapy with BRAF plus MEK inhibitors has radically changed the therapeutic landscape of melanoma, both in the advanced and the adjuvant setting. In the absence of a standardized therapeutic algorithm for BRAF mutated patients, clinicians can choose whether to start with BRAF plus MEK inhibitors or with immunotherapy, on the basis of the experience of their center, characteristics of the patient (i.e., his compliance with treatment, concomitant pathologies), and characteristics of the disease (i.e., tumor burden, LDH level).
Despite the many advances made in the therapy of these patients and the exciting results achieved, some issues remain unanswered.

Among all, one the most important is the identification and overcoming of primary and acquired resistances. Numerous drugs are currently being tested attempting to extend the pharmacological inhibition beyond MAPK pathway targeting parallel pathways molecules. Moreover, having ascertained that some forms of resistance involve epigenetic and transient mechanisms, the rechallenge with BRAF plus MEK inhibitors in resistant patients, progressing after a subsequent line (e.g., immunotherapy), is being further investigated. Furthermore, the data from clinical trials with combinations of targeted drugs and immunotherapy will be extremely interesting, especially in patients with complex clinical situations such as elevated baseline $\mathrm{LDH}$ and brain metastases.

Lastly, an important issue that needs further investigation is the treatment beyond progression, a therapeutic strategy that allows to continue the same systemic treatment in case of local progression, which could be managed with local treatments such as surgery or radiotherapy, providing that patient has a good Eastern Cooperative Oncology Group (ECOG) Performance Status and good tolerance to targeted therapy. This therapeutic strategy, commonly accepted in other solid cancers (Kuczynski et al., 2013), is still debated in melanoma. However, a retrospective study published in 2014 suggested that it may be appropriate to consider treatment beyond progression, postponing the start of a new line of therapy without evident detrimental effects (Chan et al., 2014). Similar results were achieved in another retrospective series (Scholtens et al., 2015). Currently, a study promoted by the Italian Melanoma Intergroup (IMI) aimed to prospectively assess the clinical impact of treatment beyond progression with vemurafenib plus cobimetinib, is recruiting patients in 12 Italian Centers (NCT03514901) (ClinicalTrials.gov, 2020).

This consideration underlines the importance of proceeding with both vertical and horizontal inhibition, blocking MAPK pathway but also other molecular pathways like PI3K/mTOR.

\section{AUTHOR CONTRIBUTIONS}

ET and IV contributed equally in conceiving the review focus, conducting the literature review, summarizing the manuscript, writing the first draft, and finalizing the manuscript. PG and FS designed and directed the review. $\mathrm{AB}, \mathrm{VA}, \mathrm{WB}, \mathrm{PG}$, and FS revised and made corrections to the manuscript. All authors have read and agreed to the final version of the manuscript.

\section{FUNDING}

We acknowledge the funds granted by the Italian Ministry of Health RF-2016-02362288 and Ricerca Corrente to Ospedale Policlinico San Martino, University of Genoa FRA 2017. 


\section{REFERENCES}

Ascierto, P. A., Dummer, R., Gogas, H. J., Flaherty, K. T., Arance, A., Mandala, M., et al. (2020). Update on tolerability and overall survival in COLUMBUS: landmark analysis of a randomised phase 3 trial of encorafenib plus binimetinib vs vemurafenib or encorafenib in patients with BRAF V600-mutant melanoma. Eur. J. Cancer 126, 33-44. doi: 10.1016/j.ejca.2019.11.016

Atkinson, V., Sandhu, S., Hospers, G., Long, G. V., Aglietta, M., Ferrucci, P. F., et al. (2020). Dabrafenib plus trametinib is effective in the treatment of BRAF V600mutated metastatic melanoma patients: analysis of patients from the dabrafenib plus trametinib Named Patient Program (DESCRIBE II). Melanoma Res. 30, 261-267. doi: 10.1097/CMR.0000000000000654

Boni, A., Cogdill, A. P., Dang, P., Udayakumar, D., Njauw, C.-N. J., Sloss, C. M., et al. (2010). Selective BRAFV600E inhibition enhances T-cell recognition of melanoma without affecting lymphocyte function. Cancer Res. 70, 5213-5219. doi: 10.1158/0008-5472.CAN-10-0118

Callahan, M. K., Rampal, R., Harding, J. J., Klimek, V. M., Chung, Y. R., Merghoub, T., et al. (2012). Progression of RAS-mutant leukemia during RAF inhibitor treatment. N. Engl. J. Med. 367, 2316-2321. doi: 10.1056/NEJMoa1208958

Carlino, M. S., Todd, J. R., Gowrishankar, K., Mijatov, B., Pupo, G. M., Fung, C., et al. (2014). Differential activity of MEK and ERK inhibitors in BRAF inhibitor resistant melanoma. Mol. Oncol. 8, 544-554. doi: 10.1016/j.molonc.2014.01.003

Chan, M. M. K., Haydu, L. E., Menzies, A. M., Azer, M. W. F., Klein, O., Lyle, M., et al. (2014). The nature and management of metastatic melanoma after progression on BRAF inhibitors: effects of extended BRAF inhibition. Cancer 120, 3142-3153. doi: 10.1002/cncr.28851

Chapman, P. B., Hauschild, A., Robert, C., Haanen, J. B., Ascierto, P., Larkin, J., et al. (2011). Improved survival with vemurafenib in melanoma with BRAF V600E mutation. N. Engl. J. Med. 364, 2507-2516. doi: 10.1056/ NEJMoa1103782

Chapman, P. B., Robert, C., Larkin, J., Haanen, J. B., Ribas, A., Hogg, D., et al. (2017). Vemurafenib in patients with BRAFV600 mutation-positive metastatic melanoma: final overall survival results of the randomized BRIM-3 study. Ann. Oncol. 28, 2581-2587. doi: 10.1093/annonc/mdx339

ClinicalTrials.gov (2020). Available online at: https://clinicaltrials.gov/ (Accessed January 25, 2020).

Dalle, S., Poulalhon, N., Debarbieux, S., and Thomas, L. (2013). Second primary melanomas on treatment with vemurafenib. Br. J. Dermatol. 168, 887-888. doi: $10.1111 /$ bjd.12093

Davies, M. A., Liu, P., McIntyre, S., Kim, K. B., Papadopoulos, N., Hwu, W.-J., et al. (2011). Prognostic factors for survival in melanoma patients with brain metastases. Cancer 117, 1687-1696. doi: 10.1002/cncr.25634

Davies, M. A., Saiag, P., Robert, C., Grob, J.-J., Flaherty, K. T., Arance, A., et al. (2017). Dabrafenib plus trametinib in patients with BRAFV600-mutant melanoma brain metastases (COMBI-MB): a multicentre, multicohort, openlabel, phase 2 trial. Lancet Oncol. 18, 863-873. doi: 10.1016/S1470-2045(17) 30429-1

Dréno, B., Ascierto, P. A., Atkinson, V., Liszkay, G., Maio, M., Mandalà, M., et al. (2018). Health-related quality of life impact of cobimetinib in combination with vemurafenib in patients with advanced or metastatic BRAFV600 mutationpositive melanoma. Br. J. Cancer 118, 777-784. doi: 10.1038/bjc.2017.488

Dummer, R., Ascierto, P. A., Gogas, H., Arance, A., Mandala, M., Liszkay, G., et al. (2017). Results of COLUMBUS Part 2: a phase 3 trial of encorafenib (ENCO) plus binimetinib (BINI) versus ENCO in BRAF-mutant melanoma. Ann. Oncol. 28, v428-v448. doi: 10.1093/annonc/mdx377.002

Dummer, R., Ascierto, P. A., Gogas, H. J., Arance, A., Mandala, M., Liszkay, G., et al. (2018). Encorafenib plus binimetinib versus vemurafenib or encorafenib in patients with BRAF-mutant melanoma (COLUMBUS): a multicentre, openlabel, randomised phase 3 trial. Lancet Oncol. 19, 603-615. doi: 10.1016/S14702045(18)30142-6

Eisen, T., Ahmad, T., Flaherty, K. T., Gore, M., Kaye, S., Marais, R., et al. (2006). Sorafenib in advanced melanoma: a Phase II randomised discontinuation trial analysis. Br. J. Cancer 95, 581-586. doi: 10.1038/sj.bjc.6603291

Flaherty, K. T., Infante, J. R., Daud, A., Gonzalez, R., Kefford, R. F., Sosman, J., et al. (2012). Combined BRAF and MEK inhibition in melanoma with BRAF V600 mutations. N. Engl. J. Med. 367, 1694-1703. doi: 10.1056/NEJMoa121 0093
Frederick, D. T., Piris, A., Cogdill, A. P., Cooper, Z. A., Lezcano, C., Ferrone, C. R., et al. (2013). BRAF inhibition is associated with enhanced melanoma antigen expression and a more favorable tumor microenvironment in patients with metastatic melanoma. Clin. Cancer Res. 19, 1225-1231. doi: 10.1158/1078-0432. CCR-12-1630

Gaestel, M. (2006). MAPKAP kinases - MKs - two's company, three's a crowd. Nature reviews. Mol. Cell Biol. 7, 120-130. doi: 10.1038/nrm1834

The Cancer Genome Atlas Network (2015). Genomic classification of cutaneous melanoma. Cell 161, 1681-1696. doi: 10.1016/j.cell.2015.05.044

Gibney, G. T., Messina, J. L., Fedorenko, I. V., Sondak, V. K., and Smalley, K. S. M. (2013). Paradoxical oncogenesis-the long-term effects of BRAF inhibition in melanoma. Nat. Rev. Clin. Oncol. 10, 390-399. doi: 10.1038/nrclinonc.2013.83

Global Cancer Observatory (2019). Available online at: http://gco.iarc.fr/ (accessed November 23, 2019).

González-Cao, M., Rodón, J., Karachaliou, N., Sánchez, J., Santarpia, M., Viteri, S., et al. (2015). Other targeted drugs in melanoma. Ann. Transl. Med. 3:266. doi: 10.3978/j.issn.2305-5839.2015.08.12

Grob, J. J., Amonkar, M. M., Karaszewska, B., Schachter, J., Dummer, R., Mackiewicz, A., et al. (2015). Comparison of dabrafenib and trametinib combination therapy with vemurafenib monotherapy on health-related quality of life in patients with unresectable or metastatic cutaneous BRAF Val600mutation-positive melanoma (COMBI-v): results of a phase 3, open-label, randomised trial. Lancet Oncol. 16, 1389-1398. doi: 10.1016/S1470-2045(15) 00087-X

Hauschild, A., Agarwala, S. S., Trefzer, U., Hogg, D., Robert, C., Hersey, P., et al. (2009). Results of a phase III, randomized, placebo-controlled study of sorafenib in combination with carboplatin and paclitaxel as second-line treatment in patients with unresectable stage III or stage IV melanoma. J. Clin. Oncol. 27, 2823-2830. doi: 10.1200/JCO.2007.15.7636

Hauschild, A., Dummer, R., Schadendorf, D., Santinami, M., Atkinson, V., Mandalà, M., et al. (2018). Longer Follow-Up Confirms Relapse-Free Survival Benefit With Adjuvant Dabrafenib Plus Trametinib in Patients With Resected BRAF V600-Mutant Stage III Melanoma. J. Clin. Oncol. 36, 3441-3449. doi: 10.1200/JCO.18.01219

Hauschild, A., Grob, J.-J., Demidov, L. V., Jouary, T., Gutzmer, R., Millward, M., et al. (2012). Dabrafenib in BRAF-mutated metastatic melanoma: a multicentre, open-label, phase 3 randomised controlled trial. Lancet 380, 358-365. doi: 10. 1016/S0140-6736(12)60868-X

Heidorn, S. J., Milagre, C., Whittaker, S., Nourry, A., Niculescu-Duvas, I., Dhomen, N., et al. (2010). Kinase-dead BRAF and oncogenic RAS cooperate to drive tumor progression through CRAF. Cell 140, 209-221. doi: 10.1016/j.cell.2009. 12.040

Kakavand, H., Wilmott, J. S., Menzies, A. M., Vilain, R., Haydu, L. E., Yearley, J. H., et al. (2015). PD-L1 Expression and Tumor-Infiltrating Lymphocytes Define Different Subsets of MAPK Inhibitor-Treated Melanoma Patients. Clin. Cancer Res. 21, 3140-3148. doi: 10.1158/1078-0432.CCR-14-2023

Khalili, J. S., Liu, S., Rodríguez-Cruz, T. G., Whittington, M., Wardell, S., Liu, C., et al. (2012). Oncogenic BRAF(V600E) promotes stromal cell-mediated immunosuppression via induction of interleukin-1 in melanoma. Clin. Cancer Res. 18, 5329-5340. doi: 10.1158/1078-0432.CCR-12-1632

King, A. J., Arnone, M. R., Bleam, M. R., Moss, K. G., Yang, J., Fedorowicz, K. E., et al. (2013). Dabrafenib; preclinical characterization, increased efficacy when combined with trametinib, while BRAF/MEK tool combination reduced skin lesions. PLoS One 8:e67583. doi: 10.1371/journal.pone.0067583

Korn, E. L., Liu, P.-Y., Lee, S. J., Chapman, J.-A. W., Niedzwiecki, D., Suman, V. J., et al. (2008). Meta-Analysis of Phase II Cooperative Group Trials in Metastatic Stage IV Melanoma to Determine Progression-Free and Overall Survival Benchmarks for Future Phase II Trials. JCO 26, 527-534. doi: 10.1200/ JCO.2007.12.7837

Koya, R. C., Mok, S., Otte, N., Blacketor, K. J., Comin-Anduix, B., Tumeh, P. C., et al. (2012). BRAF inhibitor vemurafenib improves the antitumor activity of adoptive cell immunotherapy. Cancer Res. 72, 3928-3937. doi: 10.1158/00085472.CAN-11-2837

Kuczynski, E. A., Sargent, D. J., Grothey, A., and Kerbel, R. S. (2013). Drug rechallenge and treatment beyond progression-implications for drug resistance. Nat. Rev. Clin. Oncol. 10, 571-587. doi: 10.1038/nrclinonc. 2013.158 
Larkin, J., Ascierto, P. A., Dréno, B., Atkinson, V., Liszkay, G., Maio, M., et al. (2014). Combined vemurafenib and cobimetinib in BRAF-mutated melanoma. N. Engl. J. Med. 371, 1867-1876. doi: 10.1056/NEJMoa1408868

Latimer, N. R., Abrams, K. R., Amonkar, M. M., Stapelkamp, C., and Swann, R. S. (2015). Adjusting for the Confounding Effects of Treatment SwitchingThe BREAK-3 Trial: dabrafenib Versus Dacarbazine. Oncologist 20, 798-805. doi: 10.1634/theoncologist.2014-0429

Long, G. V., Flaherty, K. T., Stroyakovskiy, D., Gogas, H., Levchenko, E., de Braud, F., et al. (2017a). Dabrafenib plus trametinib versus dabrafenib monotherapy in patients with metastatic BRAF V600E/K-mutant melanoma: long-term survival and safety analysis of a phase 3 study. Ann. Oncol. 28, 1631-1639. doi: 10.1093/ annonc/mdx 176

Long, G. V., Hauschild, A., Santinami, M., Atkinson, V., Mandalà, M., ChiarionSileni, V., et al. (2017b). Adjuvant Dabrafenib plus Trametinib in Stage III BRAF-Mutated Melanoma. N. Engl. J. Med. 377, 1813-1823. doi: 10.1056/ NEJMoa1708539

Long, G. V., and Margolin, K. A. (2020). Multidisciplinary approach to brain metastasis from melanoma: the emerging role of systemic therapies. Am. Soc. Clin. Oncol. Educ. Book 2013, 393-398.

Long, G. V., Stroyakovskiy, D., Gogas, H., Levchenko, E., de Braud, F., Larkin, J., et al. (2014). Combined BRAF and MEK inhibition versus BRAF inhibition alone in melanoma. N. Engl. J. Med. 371, 1877-1888. doi: 10.1056/ NEJMoa1406037

Long, G. V., Stroyakovskiy, D., Gogas, H., Levchenko, E., de Braud, F., Larkin, J., et al. (2015). Dabrafenib and trametinib versus dabrafenib and placebo for Val600 BRAF-mutant melanoma: a multicentre, double-blind, phase 3 randomised controlled trial. Lancet 386, 444-451. doi: 10.1016/S0140-6736(15) 60898-4

Long, G. V., Trefzer, U., Davies, M. A., Kefford, R. F., Ascierto, P. A., Chapman, P. B., et al. (2012). Dabrafenib in patients with Val600Glu or Val600Lys BRAFmutant melanoma metastatic to the brain (BREAK-MB): a multicentre, openlabel, phase 2 trial. Lancet Oncol. 13, 1087-1095. doi: 10.1016/S1470-2045(12) 70431-X

Long, G. V., Weber, J. S., Infante, J. R., Kim, K. B., Daud, A., Gonzalez, R., et al. (2016). Overall Survival and Durable Responses in Patients With BRAF V600-Mutant Metastatic Melanoma Receiving Dabrafenib Combined With Trametinib. J. Clin. Oncol. 34, 871-878. doi: 10.1200/JCO.2015. 62.9345

Lovly, C. M., Dahlman, K. B., Fohn, L. E., Su, Z., Dias-Santagata, D., Hicks, D. J., et al. (2012). Routine multiplex mutational profiling of melanomas enables enrollment in genotype-driven therapeutic trials. PLoS One 7:e35309. doi: 10. 1371/journal.pone.0035309

Maio, M., Lewis, K., Demidov, L., Mandalà, M., Bondarenko, I., Ascierto, P. A., et al. (2018). Adjuvant vemurafenib in resected, BRAFV600 mutationpositive melanoma (BRIM8): a randomised, double-blind, placebo-controlled, multicentre, phase 3 trial. Lancet Oncol. 19, 510-520. doi: 10.1016/S14702045(18)30106-2

Manzano, J. L., Layos, L., Bugés, C., de Los Llanos Gil, M., Vila, L., MartínezBalibrea, E., et al. (2016). Resistant mechanisms to BRAF inhibitors in melanoma. Ann. Transl. Med. 4:237. doi: 10.21037/atm.2016.06.07

Mcarthur, G., Maio, M., Arance, A., Nathan, P., Blank, C., Avril, M.-F., et al. (2016). Vemurafenib in Metastatic melanoma patients with brain metastases: an Open-Label, Single-Arm, Phase 2, Multicentre Study. Ann. Oncol. 28, 634-641. doi: 10.1093/annonc/mdw641

McArthur, G. A., Chapman, P. B., Robert, C., Larkin, J., Haanen, J. B., Dummer, R., et al. (2014). Safety and efficacy of vemurafenib in BRAFV600E and BRAFV600K mutation-positive melanoma (BRIM-3): extended follow-up of a phase 3, randomised, open-label study. Lancet Oncol. 15, 323-332. doi: 10.1016/ S1470-2045(14)70012-9

Menzies, A., Haydu, L., Visintin, L., Carlino, M., Howle, J., Thompson, J., et al. (2012). Distinguishing Clinicopathologic Features of Patients with V600E and V600K BRAF-Mutant Metastatic Melanoma. Clin. Cancer Res. 18, 3242-3249. doi: 10.1158/1078-0432.CCR-12-0052

Michielin, O., van Akkooi, A. C. J., Ascierto, P. A., Dummer, R., Keilholz, U., and Esmo Guidelines Committee. (2019). Electronic address: clinicalguidelines@esmo.org. Cutaneous melanoma: ESMO Clinical Practice Guidelines for diagnosis, treatment and follow-up†. Ann. Oncol. 30, 1884-1901. doi: 10.1093/annonc/mdz411
Minor, D. R., Puzanov, I., Callahan, M. K., Hug, B. A., and Hoos, A. (2015). Severe gastrointestinal toxicity with administration of trametinib in combination with dabrafenib and ipilimumab. Pigment Cell Melanoma Res. 28, 611-612. doi: $10.1111 /$ pcmr.12383

Morris, E. J., Jha, S., Restaino, C. R., Dayananth, P., Zhu, H., Cooper, A., et al. (2013). Discovery of a novel ERK inhibitor with activity in models of acquired resistance to BRAF and MEK inhibitors. Cancer Discov. 3, 742-750. doi: 10. 1158/2159-8290.CD-13-0070

Nazarian, R., Shi, H., Wang, Q., Kong, X., Koya, R. C., Lee, H., et al. (2010). Melanomas acquire resistance to B-RAF(V600E) inhibition by RTK or N-RAS upregulation. Nature 468, 973-977. doi: 10.1038/nature09626

Nissan, M. H., Rosen, N., and Solit, D. B. (2013). ERK pathway inhibitors: how low should we go? Cancer Discov. 3, 719-721. doi: 10.1158/2159-8290.CD-13-0245

Pasquali, S., Hadjinicolaou, A. V., Chiarion Sileni, V., Rossi, C. R., and Mocellin, S. (2018). Systemic treatments for metastatic cutaneous melanoma. Cochrane Database. Syst. Rev. 2:CD011123. doi: 10.1002/14651858.CD011123.pub2

Poulikakos, P. I., Zhang, C., Bollag, G., Shokat, K. M., and Rosen, N. (2010). RAF inhibitors transactivate RAF dimers and ERK signalling in cells with wild-type BRAF. Nature 464, 427-430. doi: 10.1038/nature08902

Queirolo, P., and Spagnolo, F. (2017). BRAF plus MEK-targeted drugs: a new standard of treatment for BRAF-mutant advanced melanoma. Cancer Metastasis Rev. 36, 35-42. doi: 10.1007/s10555-017-9660-6

Reschke, R., Simon, J.-C., and Ziemer, M. (2019). Rechallenge of targeted therapy in metastatic melanoma. J. Dtsch. Dermatol. Ges. 17, 483-486. doi: 10.1111/ddg. 13766

Ribas, A., Gonzalez, R., Pavlick, A., Hamid, O., Gajewski, T. F., Daud, A., et al. (2014). Combination of vemurafenib and cobimetinib in patients with advanced BRAF(V600)-mutated melanoma: a phase 1b study. Lancet Oncol. 15, 954-965. doi: 10.1016/S1470-2045(14)70301-8

Ribas, A., Hodi, F., Lawrence, D., Atkinson, V., Starodub, A., Carlino, M., et al. (2016). Pembrolizumab (pembro) in combination with dabrafenib (D) and trametinib (T) for BRAF - mutant advanced melanoma: Phase 1 KEYNOTE-022 study. J. Clin. Oncol. 34, 3014-3014. doi: 10.1200/JCO.2016.34.15_suppl.3014

Ribas, A., Hodi, F. S., Callahan, M., Konto, C., and Wolchok, J. (2013). Hepatotoxicity with combination of vemurafenib and ipilimumab. N. Engl. J. Med. 368, 1365-1366. doi: 10.1056/NEJMc1302338

Robarge, K., Schwarz, J., Blake, J., Burkard, M., Chan, J., Chen, H., et al. (2014). Abstract DDT02-03: discovery of GDC-0994, a potent and selective ERK1/2 inhibitor in early clinical development. Cancer Res. 74, DDT02-03. doi: 10.1158/ 1538-7445.AM2014-DDT02-03

Robert, C., Grob, J. J., Stroyakovskiy, D., Karaszewska, B., Hauschild, A., Levchenko, E., et al. (2019). Five-Year Outcomes with Dabrafenib plus Trametinib in Metastatic Melanoma. N. Engl. J. Med. 381, 626-636. doi: 10. 1056/NEJMoa1904059

Robert, C., Karaszewska, B., Schachter, J., Rutkowski, P., Mackiewicz, A., Stroiakovski, D., et al. (2015). Improved overall survival in melanoma with combined dabrafenib and trametinib. N. Engl. J. Med. 372, 30-39. doi: 10.1056/ NEJMoa1412690

Robert, C., Karaszewska, B., Schachter, J., Rutkowski, P., Mackiewicz, A., Stroyakovskiy, D., et al. (2016). Three-year estimate of overall survival in COMBI-v, a randomized phase 3 study evaluating first-line dabrafenib (D) + trametinib $(\mathrm{T})$ in patients (pts) with unresectable or metastatic BRAF V600E/K-mutant cutaneous melanoma. Ann. Oncol. 27, 1-36. doi: 10.1093/ annonc/mdw 435.37

Rubinstein, J. C., Sznol, M., Pavlick, A. C., Ariyan, S., Cheng, E., Bacchiocchi, A., et al. (2010). Incidence of the V600K mutation among melanoma patients with BRAF mutations, and potential therapeutic response to the specific BRAF inhibitor PLX4032. J. Transl. Med. 8:67. doi: 10.1186/1479-58 76-8-67

Sanchez-Vega, F., Mina, M., Armenia, J., Chatila, W. K., Luna, A., La, K. C., et al. (2018). Oncogenic Signaling pathways in the cancer genome atlas. Cell 173:321-337.e10. doi: 10.1016/j.cell.2018.03.035

Sapkota, B., Hill, C. E., and Pollack, B. P. (2013). Vemurafenib enhances MHC induction in BRAFV600E homozygous melanoma cells. Oncoimmunology 2:e22890. doi: 10.4161/onci.22890

Schadendorf, D., Amonkar, M. M., Stroyakovskiy, D., Levchenko, E., Gogas, H., de Braud, F., et al. (2015). Health-related quality of life impact in a randomised phase III study of the combination of dabrafenib and trametinib versus 
dabrafenib monotherapy in patients with BRAF V600 metastatic melanoma. Eur. J. Cancer 51, 833-840. doi: 10.1016/j.ejca.2015.03.004

Scholtens, A., Geukes Foppen, M. H., Blank, C. U., van Thienen, J. V., van Tinteren, H., and Haanen, J. B. (2015). Vemurafenib for BRAF V600 mutated advanced melanoma: results of treatment beyond progression. Eur. J. Cancer 51, 642-652. doi: 10.1016/j.ejca.2015.01.009

Shi, H., Hugo, W., Kong, X., Hong, A., Koya, R. C., Moriceau, G., et al. (2014). Acquired resistance and clonal evolution in melanoma during BRAF inhibitor therapy. Cancer Discov. 4, 80-93. doi: 10.1158/2159-8290.CD-13-0642

Society for Melanoma Research 2016 Congress (2017). Pigment Cell \& Melanoma Research, Vol. 30. Hoboken, NJ: Wiley, 76-156. doi: 10.1111/pcmr.12547

Society for Melanoma Research Congress (2019). Available online at: https://www. societymelanomaresearch.org/congress (accessed January 26, 2020).

Spagnolo, F., Boutros, A., Tanda, E., and Queirolo, P. (2019). The adjuvant treatment revolution for high-risk melanoma patients. Semin. Cancer Biol. 59, 283-289. doi: 10.1016/j.semcancer.2019.08.024

Spagnolo, F., Ghiorzo, P., Orgiano, L., Pastorino, L., Picasso, V., Tornari, E., et al. (2015). BRAF-mutant melanoma: treatment approaches, resistance mechanisms, and diagnostic strategies. Oncol. Targets Ther. 8, 157-168. doi: 10.2147/OTT.S39096

Spagnolo, F., Picasso, V., Lambertini, M., Ottaviano, V., Dozin, B., and Queirolo, P. (2016). Survival of patients with metastatic melanoma and brain metastases in the era of MAP-kinase inhibitors and immunologic checkpoint blockade antibodies: a systematic review. Cancer Treat. Rev. 45, 38-45. doi: 10.1016/j. ctrv.2016.03.003

Straussman, R., Morikawa, T., Shee, K., Barzily-Rokni, M., Qian, Z. R., Du, J., et al. (2012). Tumour micro-environment elicits innate resistance to RAF inhibitors through HGF secretion. Nature 487, 500-504. doi: 10.1038/nature11183

Su, F., Viros, A., Milagre, C., Trunzer, K., Bollag, G., Spleiss, O., et al. (2012). RAS mutations in cutaneous squamous-cell carcinomas in patients treated with BRAF inhibitors. N. Engl. J. Med. 366, 207-215. doi: 10.1056/NEJMoa1105358

Van Allen, E. M., Wagle, N., Sucker, A., Treacy, D. J., Johannessen, C. M., Goetz, E. M., et al. (2014). The genetic landscape of clinical resistance to RAF inhibition in metastatic melanoma. Cancer Discov. 4, 94-109. doi: 10.1158/2159-8290.CD13-0617

Vanni, I., Tanda, E. T., Spagnolo, F., Andreotti, V., Bruno, W., and Ghiorzo, P. (2020). The current state of molecular testing in the BRAF-Mutated melanoma landscape. Front. Mol. Biosci. 7:113. doi: 10.3389/fmolb.2020.00113

Villanueva, J., Vultur, A., Lee, J. T., Somasundaram, R., Fukunaga-Kalabis, M., Cipolla, A. K., et al. (2010). Acquired resistance to BRAF inhibitors mediated by a RAF kinase switch in melanoma can be overcome by cotargeting MEK and IGF-1R/PI3K. Cancer Cell 18, 683-695. doi: 10.1016/j.ccr.2010. 11.023

Wilson, T. R., Fridlyand, J., Yan, Y., Penuel, E., Burton, L., Chan, E., et al. (2012). Widespread potential for growth-factor-driven resistance to anticancer kinase inhibitors. Nature 487, 505-509. doi: 10.1038/nature11249

Wong, D. J. L., Robert, L., Atefi, M. S., Lassen, A., Avarappatt, G., Cerniglia, M., et al. (2014). Antitumor activity of the ERK inhibitor SCH772984 [corrected] against BRAF mutant. NRAS mutant and wild-type melanoma. Mol. Cancer 13:194. doi: 10.1186/1476-4598-13-194

Zimmer, L., Hillen, U., Livingstone, E., Lacouture, M. E., Busam, K., Carvajal, R. D., et al. (2012). Atypical melanocytic proliferations and new primary melanomas in patients with advanced melanoma undergoing selective BRAF inhibition. J. Clin. Oncol. 30, 2375-2383. doi: 10.1200/JCO.2011.41.1660

Conflict of Interest: The authors declare that the research was conducted in the absence of any commercial or financial relationships that could be construed as a potential conflict of interest.

Copyright (c) 2020 Tanda, Vanni, Boutros, Andreotti, Bruno, Ghiorzo and Spagnolo. This is an open-access article distributed under the terms of the Creative Commons Attribution License (CC BY). The use, distribution or reproduction in other forums is permitted, provided the original author(s) and the copyright owner(s) are credited and that the original publication in this journal is cited, in accordance with accepted academic practice. No use, distribution or reproduction is permitted which does not comply with these terms. 